\title{
Molecular level correlation between probable homoeopathic medicines and bio-samples of patients
}

\begin{abstract}
Background: Homoeopathy is based on the principle that 'like cures like' - substances capable of causing disorder in healthy subjects are used as medicines to treat similar patterns of disorder in sick persons. It is similarity at macroscopic level. This article aims to find out whether any similarity picture exists in molecular level also, that can make selection of medicines based on physically measurable paramters.

Method: This investigation involved three arthritis patients. It consisted of getting frequency domain signatures for dielectric functions of (i) medicines selected for them on the basis of macroscopic similarity and (ii) bio-fluids (blood serums) of the patients. Then, they were compared to find existence/absence of similarity between them.

Results: Medicines administered to the three patients were Guaiacum 30c, Sulphur 200c and Natrum Mur 30c. Dielectric function resonance frequencies for these medicines were $22.9 \mathrm{MHz}, 23.5 \mathrm{MHz}$ and $23.8 \mathrm{MHz}$, whereas the bio-fluids of the patients had $22.5 \mathrm{MHz}$, $23.2 \mathrm{MHz}$ and $24.9 \mathrm{MHz}$ respectively.

Conclusion: It was found that similarity (resonance frequency matching) existed in spectral signatures of the two groups of substances i.e. bio-fluids (blood serum) and the indicated remedies for the first two patients who got beneficial results with their medicines. This similarity was not evident in the third patient where medicine failed to act and the patient was not benefited. These results are quite encouraging. It seems to be an indication of validity of Homoeopathic Principle at molecular level too, opening a new chapter of fundamental research in medicine.
\end{abstract}

Keywords: dielectric functions, resonance frequency matching, spectral signatures, homoeopathic principle at the molecular level
Volume 3 Issue 4 - 2016

\author{
Rajat Chattopadhyay,' Chitta Ranjan Mahata' \\ 'MBHMC \& Hospital, India \\ ${ }^{2}$ Indian Institute of Engineering Science and Technology, India
}

Correspondence: Chitta Ranjan Mahata, Hony Emeritus Scientist, IIEST, Flat-C2, 50/I College Road, Howrah-7I I I03,WB, Shibpur, India,Tel 919433739180, Email crmahata@gmail.com

Received: December 17, 2015 | Published: March 23, 2016

\section{Introduction}

Macroscopic effects of medicines in healthy individuals (while proving) are matched with macroscopic manifestations of disease symptoms for selection of medicines for treating a patient. This is the principle followed in Homoeopathy. This kind of correlation does not have quantified measurable parameters of physical science. The questions we asked ourselves are: Can we have such correlation at molecular level? How to validate or reject it? For advancing to this goal we noted that the most promising hypothesis for explaining medicinal value of potentised as well as un-potentised substances is the 'Structural concept', which may be stated as: A substance is to be recognized as a medicine if it has the capability of curing disease(s) while its medicinal property is to be attributed to molecular structure of vehicle like water or of distinct chemical substance when it exists. ${ }^{1}$

Water structure hypothesis is also directly or indirectly supported by a number of researchers. Barnard $\mathrm{GO}^{2}$ was possibly the first to hint at the structural concept though he named it as water polymer. But, his work lacked experimental support. Stupendously successful experimental results were reported by Boyd WE. ${ }^{3}$ But, explanation for the emanations he detected by his 'emanometer' were missing at that time. Much later, Chattopadhyay R et al., ${ }^{4}$ offered an explanation suggesting that it originated from structured vehicle getting excitation from ambient electromagnetic radiation. Anagnostatos GS et al., ${ }^{5}$ proposed his structural model calling the structured water (of vehicle of potentisation) as 'clathrates'. He described how clathrates are likely to be generated at different stages of serial dilution. Water, with a simple chemical formula of $\mathrm{H} 2 \mathrm{O}$, has attracted the attention of many researchers for explaining its exceptional properties. It is evidenced by Chaplin $\mathrm{M}$ enlisting 2388 investigations on water. ${ }^{6}$ Attempts continue. Maity $\mathrm{T}$ et al., ${ }^{7,8}$ and Mahata $\mathrm{CR}^{9,10}$ showed experimentally that water structures were present in medicines both below and above the Avogadro limit of 12c. Roy Rustum et al., ${ }^{11,12}$ firmly supports the structural concept with some experimental results. Davenas E et al., ${ }^{13}$ in their experiments with human basophile degranulation show that 'memory' of the original substance are retained in serial dilution followed by vortexing even beyond the Avogadro limit. It was not a work with medicines but it has relevance to the dilution/ potentisation of homoeopathy. They called it as 'water memory', but we like to call it induced structure. Similarly, Rey Louis ${ }^{14}$ in his investigation with dilution of substance like $\mathrm{LiCl}$ and $\mathrm{NaCl}$ showed that the hydrogenbonded network of water remained modified even in dilutions beyond Avogadro limit. We may assume that such modification will lead to variation in structure of molecular clusters of the vehicle. Montagnier L et al., ${ }^{15}$ demonstrated that even massively diluted (1012) DNA and RNA solutions continue to emit electromagnetic radiations like the un-diluted one. Ref $^{4}$ offers a possible explanation of this phenomenon in terms of structures. Support to structural model for extremely diluted substances also comes from the work of Elia V et al., ${ }^{16}$ But, the structural model as a generalized concept was possibly first advanced by Mahata CR. ${ }^{1}$ 


\section{Method}

Investigations of Mahata $\mathrm{CR}^{9,10}$ using the technique of dielectric dispersion give stronger credence to the 'Structural concept'. This prompted us to investigate whether the macroscopic matching (that is, homoeopathic principle) can be extended to molecular level. Can we find any correlation at molecular level between probable medicines and patients' bio-samples by using the same technique? We selected medicines for several cases of arthritis. Then, we recorded frequency domain signatures for dielectric functions of these medicines and blood serum of the patients with experimental set up as shown in Figure 1.

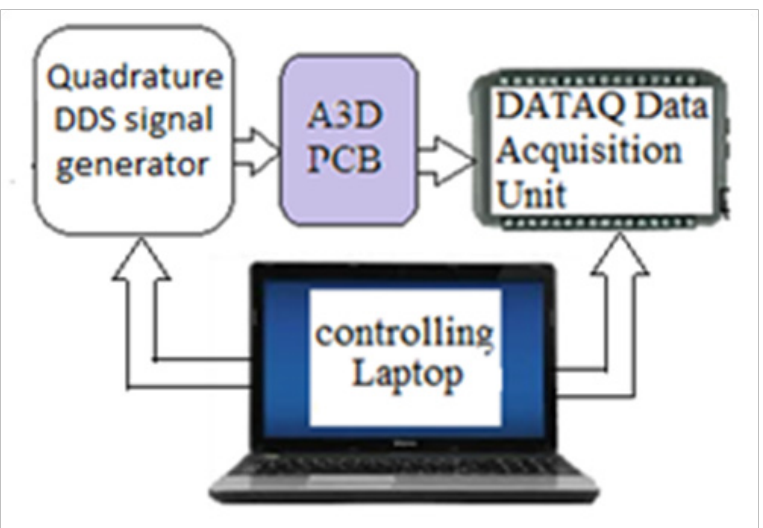

Figure I Physical sketch of the experimental set up.

The Quadrature DDS using AD9854 IC chip was manufactured in Shenzhen, China; the Data Acquisition Unit was DI-149 manufactured by WINDAQ, USA. The A3D PCB giving outputs representing real and imaginary component values of dielectric function was developed by us indigenously. Its functional diagram is shown in Figure 2.

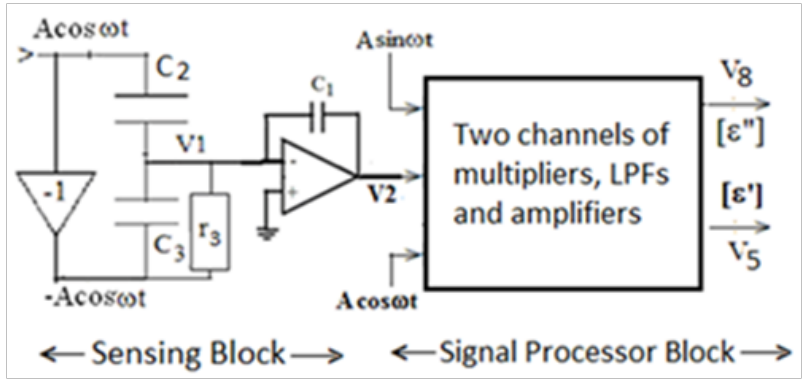

Figure 2 Functional details of the A3D PCB.

Here, $\mathrm{C} 1$ is an air capacitor. $\mathrm{C} 2$ is the capacitance of a reference cell formed by two metal plates measuring 3" $\mathrm{x} 4$ " (76.2 $\mathrm{mm}$ x $101.6 \mathrm{~mm})$ separated by $1.5 \mathrm{~mm}$, a polythene pouch serving as its dielectric material. Its capacitance is $300 \mathrm{pF}$. C3 in parallel with $\mathrm{r} 3$ represents an imperfect capacitor also formed by two metal plates as for $\mathrm{C} 1$, but its dielectric material comprised of the test material, namely sugar globules (six in number of 10 no.size) soaked in medicine or biosample of subject (as the case may be) and then dried and finally dissolved in $5 \mathrm{ml}$ of water in a polythene pouch placed between the metal plates. Its capacitance was also adjusted to $300 \mathrm{pF}$ by fineadjustment of the gap between the metal plates. The frequency domain signature was taken from 1.0 MHz to $30.0 \mathrm{MHz}$. Frequency step and ramp rate of the Quadrature DDS signal generator and sampling rate of Data Acquisition unit were adjusted to get a graphical record of 1 div equal to $1 \mathrm{MHz}$. In all these records upper trace represents loss in the sample and the lower trace, dielectric constant.

\section{Results}

Patient -1: female, 40yrs, OPD registration no. H-F0453. Symptoms: pain in long bone $<$ night, repeated sore throat and mouth ulcer, syphilitic past and family history, chilly patient, deep central crack on tongue, offensive discharges. Diagnosis - osteoarthritis. Medicine prescribed - Guaiacum 30c. Resonance frequency of applied medicine is $22.9 \mathrm{MHz}$ and resonance frequency of bio-fluid of patient - 22.5MHz. Status - marked improvement (Figures 3a \& 3b).

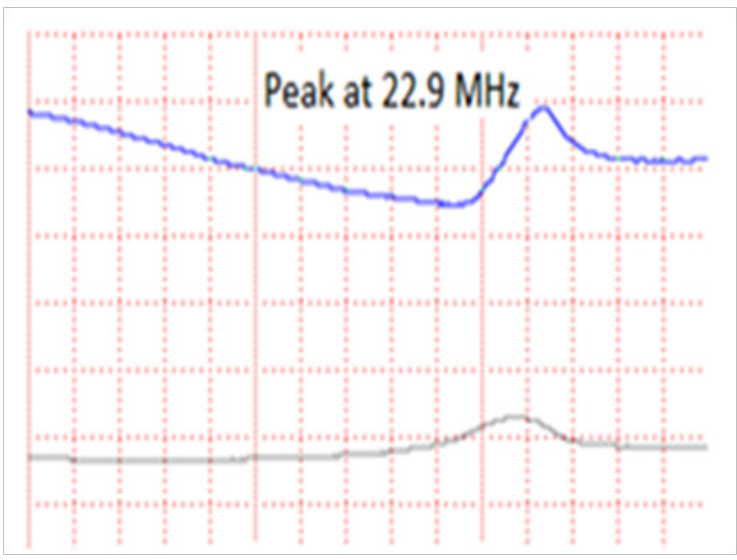

Figure 3a Spectra for Guaiacum 30c.

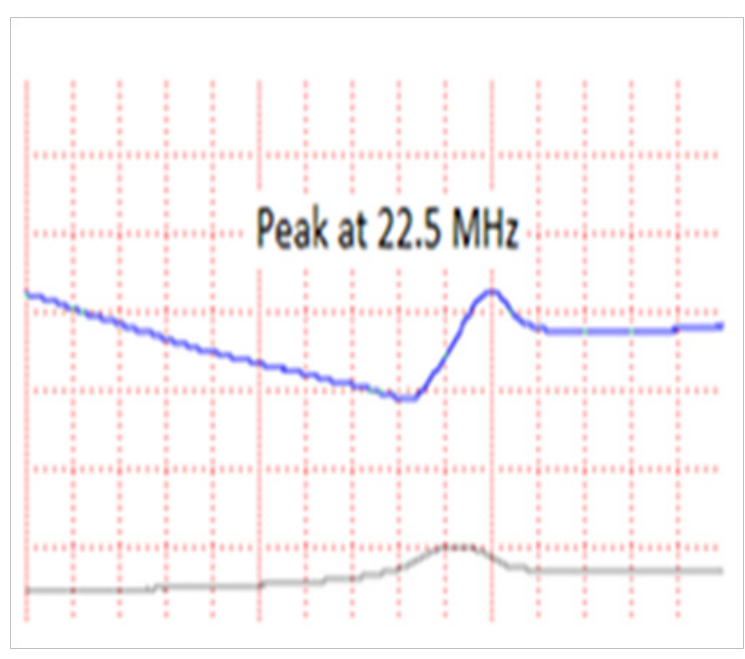

Figure 3b Spectra for bio-fluid of patient-I.

Patient -2: female, 56yrs, OPD registration no. H-F4304. Symptoms: pain in legs < standing, hunger intolerance, Past history of suppressed eruption with itching, hot patient, craving for sweets, burning sensation. Diagnosis - osteoarthritis. Medicine prescribed Sulphur 200c. Resonance frequency of applied medicine $-23.5 \mathrm{MHz}$ and resonance frequency of bio-fluid of patient - 23.2 MHz. Status -improvement (Figures 4a \& 4b).

Patient -3: female, 36yrs, OPD registration no. H-F95850. Symptoms: pain and swelling of middle and ring fingers of both hands > rest, emotional, shy, can't eat without salt, aversion to milk, sunlight aggravates, scanty menstruation. Diagnosis - seronegative rheumatoid arthritis. Medicine prescribed - Natrum Mur 30c. Resonance frequency of applied medicine $-23.8 \mathrm{MHz}$ and resonance frequency of bio-fluid of patient - 24.9 MHz. Status - some improvement at first but then no improvement (Figures 5a \& 5b). 


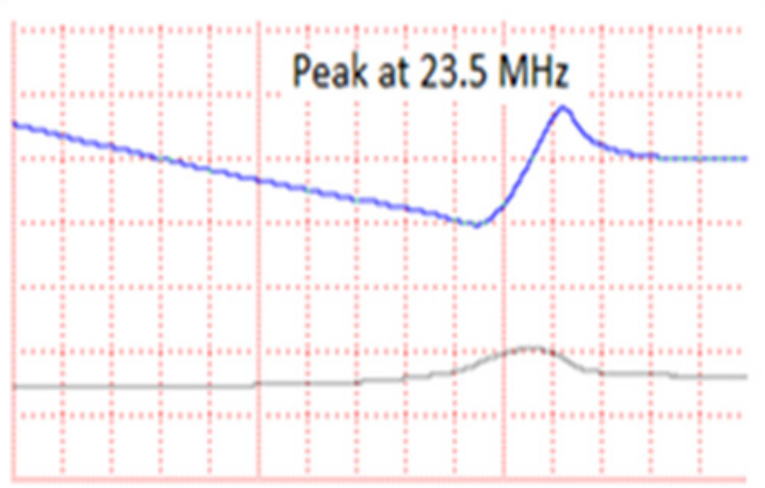

Figure 4a Spectra for Sulphur 200c.

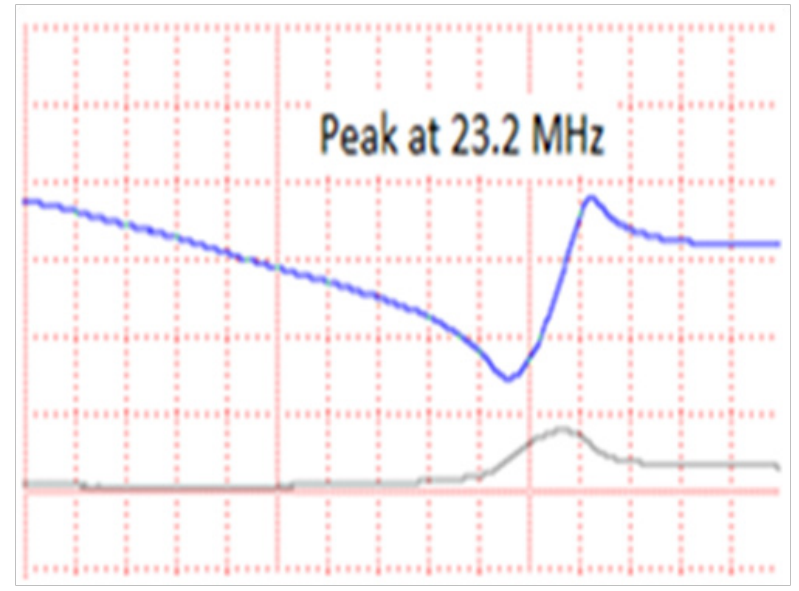

Figure 4b Spectra for bio-fluid of patient-2.

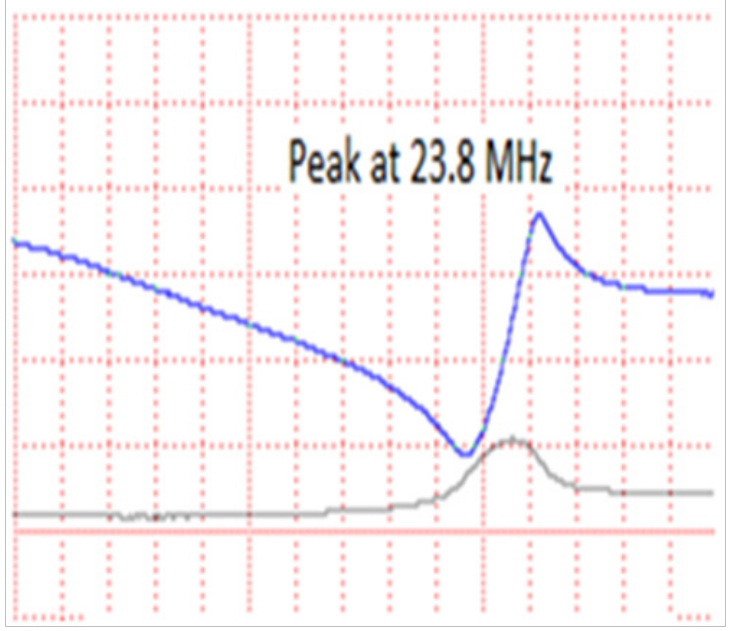

Figure 5a Spectra for Natrum Mur 30c.

\section{Discussion and Conclusion}

The first two patients had a close match between the resonance frequencies of the medicines and of the bio-fluids. Both of them were benefitted with their medicines. The third patient did not have a good matching of the resonance frequencies. She was not benefited by the medicine. Initial results are quite encouraging. It seems to be an indication of validity of Homoeopathic Principle at the molecular level too, which may open a new chapter of fundamental research in medicine and give a new tool to physicians. This research may also be extended to other bio-samples taken from different diseased organs.

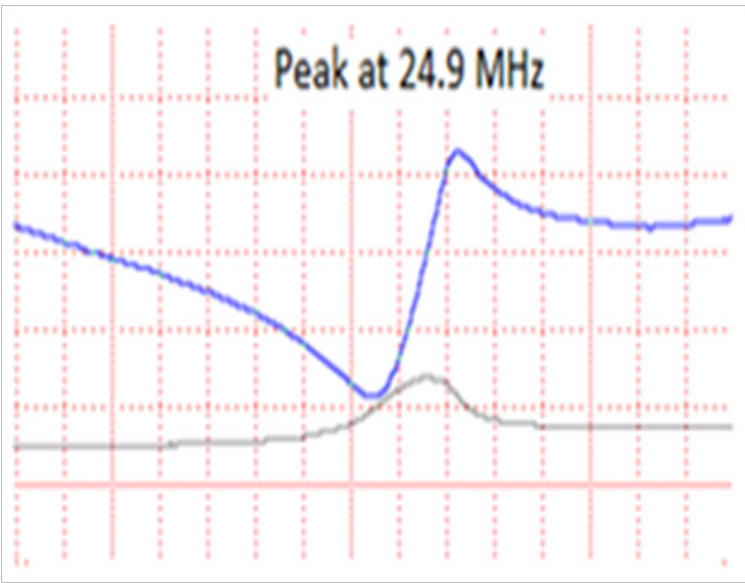

Figure 5b Spectra for bio- fluid of patient-3.

\section{Acknowledgements}

The background and infrastructural supports from MBHMC \& Hospital Howrah, its affiliating authority The WB University of Health Sciences, IIEST Shibpur and free supply of medicines and chemicals by M/S Hahnemann Publishing Co. Ltd, Kolkata in various stages of our research are gratefully acknowledged.

\section{Conflicts of interest}

Author declares there are no conflicts of interest.

\section{Funding}

None.

\section{References}

1. Mahata CR. Structural model explains high potency homoeopathic medicines and leads to a generalized understanding of medicines. Int $J$ Complement Alt Med. 2015;1(2):00011.

2. Barnard GO. Microdose paradox - a new concept. J Am Inst Homeopath. 1965;58(7):205-212.

3. The electronic reactions of Abrams Communication to the royal society of medicine by a committee of which sir thomas horder is chairman. $\mathrm{Br}$ Med J. 1924;1(3343):179-185.

4. Chattopadhyay R, Sadhukhan M, Pal A, et al. On electromagnetic signals from vastly diluted DNA solutions, potentised medicines and even metals. Wesleyan Journal of Research . 2012;5(1):50-55.

5. Anagnostatos GS, Vithoulkas G, Garzonis P, et al.A working hypothesis for homoeopathic microdiluted remedies. The Berlin Journal of Research in Homoeopathy. 1991;1(3):141-147.

6. Chaplin M. Water Structure and Science. 2015.

7. Maity Tanmoy, Ghosh D, Mahata CR. Theory and Instrumentation related to Potentised Homoeopathic Medicines. IE(I) Journal. 2007;2(3):27-31.

8. Maity T, Ghosh D, Mahata CR. Effect of Dielectric Dispersion on Potentised Homoeo-Medicines. Homeopathy. 2010;99(2):99-103.

9. Mahata CR. Dielectric Dispersion Studies Indicate Change in Structure of Water by Potentised Homeopathic Medicines. IE(I). 2013;93(4):231235 
10. Mahata CR. Dielectric Dispersion Studies of Some Potentised Homoeopathic Medicines Reveal Structured Vehicle. Homeopathy. 2013;102(4):262-267.

11. Roy R, Tiller WA, Bell I, et al. The structure of liquid water; novel insights from materials research; potential relevance to homeopathy. Materials Research Innovations. 2005;3(2):577-608.

12. Rao ML, Roy R, Bell IR, et al.The defining role of structure (including epitaxy) in the plausibility of homeopathy Homeopathy. 2007;96(3):175182.

13. Davenas E, Beauvais F, Amara J, et al. Human basophil degranulation triggered by very dilute antiserum against IgE. Nature. 1988;333:816818.
14. Rey L. Thermo-luminescence of ultra-high dilutions of lithium chloride and sodium chloride. Physica A. 2003;323:67-74.

15. Montagnier L, Aäissa J, Ferris S, et al. Electromagnetic signals are produced by aqueous nanostructures derived from bacterial DNA sequences. Interdiscip Sci. 2009;1(2):81-90.

16. Elia V, Ausanio G, Gentile F, et al. Experimental evidence of stable water nanostructures in extremely dilute solutions, at standard pressure and temperature. Homeopathy. 2014;103(4):44-50. 